\title{
Amplitude Modulation detection ability can be differentially affected using state-dependent Transcranial Magnetic
}

\author{
Stimulation
}

Abbreviated title: TMS differentially affects sensitivity to AM

* Adam M. Partridge amp530@york.ac.uk

York NeuroImaging Centre, Department of Psychology, University of York, YO10 5NY, UK

Contributions: Designed research, Performed Research, Analyzed data, Wrote the paper

Garreth Prendergast garreth.prendergast@manchester.ac.uk

Manchester Centre for Audiology and Deafness, University of Manchester, M13 9PL, UK

Contributions: Designed research, Wrote the paper

* Mark Hymers mark.hymers@ynic.york.ac.uk

York NeuroImaging Centre, Department of Psychology, University of York, YO10 5NY, UK

Contributions: Designed research, Performed Research, Analyzed data, Wrote the paper

*: Corresponding authors

Number of pages: $35+$ title page; Number of figures: 3; Number of tables: 2

Number of words: Abstract: 234; Introduction: 840; Methods: 1,789;

Results: 1,040; Discussion: 2,154

Conflicts of Interest: The authors declare no competing financial interests

A pre-print of this paper has been uploaded to Open Science Framework at

https://osf.io/twfc5/ 


\section{Abstract}

2 Temporal changes in the speech envelope are crucial for intelligibility, and humans 3 are particularly sensitive to amplitude modulations (AM) at frequencies commen4 surate with the syllabic rate $(4 \mathrm{~Hz})$. Some asymmetric models of auditory processing 5 have attempted to explain observed functional asymmetries between left and right 6 auditory cortices in terms of temporal integration windows of differing lengths. In 7 this paper we use an fMRI-localised, state-dependent, dual-pulse TMS paradigm to 8 investigate auditory processing as exemplified by detection of sinusoidal amplitude9 modulation of a broadband noise carrier. The effect of left and right hemisphere TMS on accuracy levels for detection of such modulations at rates of 4 and $40 \mathrm{~Hz}$ under conditions of behavioural adaptation were examined. Effects of TMS on AM

${ }_{22}$ Highlights

- Detection of amplitude modulation in auditory stimuli can be perturbed using TMS

- An interaction is found between hemisphere of stimulation and AM rate of the stimulus 
27

28

${ }_{29}$ Keywords

30 - Amplitude modulation

31 - Behavioural adaptation

32

33

34 considered

- Human behavioural

- fMRI

- TMS

- An explanation based on neural coding mechanisms and timing of TMS is 


\section{Introduction}

36 The structure of human speech is highly dynamic, consisting of continuous changes 37 in both amplitude and frequency content. Slow temporal amplitude modulations 38 (Rosen 1992), are known to be crucial to speech intelligibility (Drullman et al.

1994a,b). Indeed, intelligibility remains high even under conditions of severely degraded spectral content, provided that these amplitude modulations are preserved (Shannon et al. 1995).

Psychophysical work has shown that humans are particularly sensitive to detecting $\mathrm{AM}$ at rates commensurate with the speech envelope and that $\mathrm{AM}$ detection can be characterised as a low-pass (Viemeister 1979; Rees et al. 1986; Kohlrausch et al. 2000) or band-pass process (Edwards and Chang 2013). It has been proposed that AM is extracted by a bank of filters, each tuned to different modulation rates (Dau et al. 1997a,b; Jepsen et al. 2008; Simpson et al. 2013; Xiang et al. 2013). The exact anatomical locus of such a modulation filterbank is currently unclear, although it is known that progressively higher areas of the auditory pathway show peak responsivity for lower AM rates (Harms and Melcher 2002; Harms et al. 2005), behaviour which has been compared to a cascade of modulation filters (Giraud et al. 2000).

In addition to differential sensitivity from lower to higher auditory areas, several hypotheses have been proposed to account for observed asymmetries in auditory processing. One hypothesis holds that the left hemisphere preferentially processes temporal structure whilst the right hemisphere is more sensitive to spectral cues (Zatorre and Belin 2001; Zatorre et al. 2002) whilst an alternative ('Asymmetric Sampling in Time'; Poeppel 2003) proposes that the right hemisphere integrates over longer timescales $(\sim 250 \mathrm{~ms})$ than the left hemisphere $(\sim 25 \mathrm{~ms})$. The longer of these timescales $(\sim 250 \mathrm{~ms})$ corresponds closely with the syllabic rate of speech $(\sim 4 \mathrm{~Hz})$. The shorter timescale $(\sim 25 \mathrm{~ms})$ corresponds to a frequency of $40 \mathrm{~Hz}$, which is around the phonemic rate of speech. Dichotomous accounts of asymmetric pro- 
cessing have, however, been challenged - both due to a of lack of empirical support for left hemisphere temporal selectivity as well as the fact that such a division does not account for the true structure of the speech signal (McGettigan and Scott 2012).

In the current study, TMS was used to further investigate the behavioural detection of sinusoidal amplitude modulation of broadband noise. The study was designed with specific reference to asymmetric models of auditory processing.

A state-dependent, dual-pulse, TMS method was used to perturb judgements in a sinusoidal AM detection task at modulation rates of $4 \mathrm{~Hz}$ and $40 \mathrm{~Hz}$. When TMS is delivered to neuronal populations in different activation states, state-dependent differential effects have been observed (Silvanto and Pascual-Leone 2008; Silvanto 2008; Cattaneo and Silvanto 2008). In this context, state-dependent TMS essentially refers to the fact that the impact on behaviour caused by the TMS pulse is altered by the nature of the processing being performed by the neuronal population at the time at which the pulse is administered. These effects include behavioural facilitations for detection of adapted stimuli, suggesting that the common conceptualisation of TMS as a purely disruptive tool is inadequate (Romei et al. 2016). An overall framework has recently been proposed that can explain classic 'virtual lesion' inhibitory effects of TMS and state-dependent effects (Silvanto and Cattaneo 2017). Combining state-dependent TMS with behavioural adaptation is a promising method for investigating AM detection for three principal reasons. Firstly, the perceptual effects of adaptation to amplitude and frequency modulated sounds are well characterised (Regan and Tansley 1979; Tansley and Suffield 1983; Bacon and Grantham 1989; Houtgast 1989; Wojtczak and Viemeister 2005; Kleinschmidt and Jaeger 2015). Secondly, state-dependent TMS potentially allows the dissociation of cortical functioning in brain areas with overlapping populations responsible for processing different rates of AM, as may be expected based on previous localisation studies (Barton et al. 2012; Herdener et al. 2013). Finally, as stimulation is delivered immediately prior to the auditory test stimulus in the state-dependent TMS 
paradigm, potential auditory masking due to the TMS click artefact is avoided. Whilst different forms of neurostimulation has been used to modulate aspects of auditory perception such as temporal discrimination (Bueti et al. 2008; Heimrath et al. 2014), melody discrimination (Andoh and Zatorre 2011), phoneme categorisation (Rufener et al. 2016), and tinnitus (Weisz et al. 2012), to our knowledge, TMS has not previously been used to investigate AM processing.

We proposed three research questions. i) can AM detection ability be affected by TMS? ii) do these effects show state-dependency? iii) do these effects show hemispheric differences consistent with lateralised accounts of auditory processing?

Three pre-registered hypotheses were formulated (Partridge 2017). It was predicted that AM detection performance would differ between real and sham TMS conditions. Secondly, it was predicted that TMS effects would show state-dependency; i.e. performance would only be improved by TMS when the modulation rates of the adaptor and test stimuli were congruent. Finally, it was predicted that effects would show an interaction with hemisphere of stimulation such that left hemisphere stimulation would have a greater effect on $40 \mathrm{~Hz}$ AM detection performance and right hemisphere stimulation would have a greater effect on $4 \mathrm{~Hz}$ AM detection performance. 


\section{Material and Methods}

\section{Subjects}

Ten subjects ( 4 female; $\mathrm{M}=23.1, \mathrm{SD}=5.14$ ) participated in the experiment. Subjects responded to an advert and were paid or reimbursed in course credit for participation except for one participant who was a member of the research team. All subjects were TMS and MRI eligible, right-handed, native English speaking and had self-reported normal hearing. The project was approved by the Research Governance Committee, York Neuroimaging Centre, University of York and conformed to the guidelines of the Declaration of Helsinki. All participants gave written informed consent. One participant withdrew from the experiment and data from one participant had to be discarded due to acoustic noise caused by building work during the testing sessions, leaving a total of 8 participants. All data collection was undertaken after the pre-registration document (Partridge 2017) was made publicly available.

\section{Experimental Design}

\section{Audiogram}

Subjects underwent an air conductance pure tone audiogram to determine that hearing thresholds were above eligibility requirements. Audiograms were conducted using an Interacoustics AD226 audiometer (Interacoustics, Middelfart, Denmark) and Telephonics TDH-39P earphones (Telephonics, NY, USA). Subjects were excluded from further testing if they did not meet the threshold criteria of $\leq 20 \mathrm{~dB}$ HL at octave frequencies from $250-8000 \mathrm{~Hz}$ in both ears.

\section{Psychophysical Testing}

A double-walled, sound-attenuated booth was used for psychophysical testing. Auditory stimuli were presented diotically via Etymotic ER-2 headphones (Etymotic 
Research Inc., IL, USA). Following the design of Viemeister (1979), a 2-alternative forced choice (2AFC) task was used to determine psychophysical thresholds for AM detection. Each stimulus consisted of three continuous $500 \mathrm{~ms}$ periods of broadband noise (low-pass filtered at $8 \mathrm{kHz}$ ). In each trial, the first or third period was randomly chosen to be sinusoidally amplitude modulated. The second (centre) 500ms period was a reference period and always consisted of unmodulated broadband noise. Participants indicated which interval they thought contained modulation by means of a key press. Participants were tested separately at AM rates of 4 and $40 \mathrm{~Hz}$ with 20 practice trials delivered at each rate before testing began. LEDs were used to indicate the two test intervals and feedback was given after each trial. The starting phase of the modulation was drawn randomly from a uniform distribution ranging from 0 to $2 \pi$ and the standard and comparison interval power were normalised to match the RMS of the centre period. Stimuli were generated at a sample rate of 44.1 $\mathrm{kHz}$ using Python and presented using PsychoPy (Peirce 2007) and the PyAudio module ( https://people.csail.mit.edu/hubert/pyaudio/) through an EMU 0204 24-bit DAC (Creative Technology Ltd, Singapore). Stimuli were presented at $70 \mathrm{~dB}$ SPL as calibrated using an artificial ear (B\&K 4153) and a sound level meter (B\&K 2260). The AM depth of the comparison interval was systematically modified using an adaptive staircase procedure (Levitt 1971). Two interleaved staircases were used (1-up, 2-down; 1-up, 3-down). Before each trial, a random choice was made between the remaining staircases and this continued until each staircase had reached 14 reversals. A step size of $1 \mathrm{~dB}$ was used for the first 4 reversals of each staircase, which decreased to $0.5 \mathrm{~dB}$ for the next 4 reversals and to $0.25 \mathrm{~dB}$ for the final 6 reversals. Participants completed three runs (six staircases in total) at each of the 4 and $40 \mathrm{~Hz}$ modulation rates. For 4 and $40 \mathrm{~Hz}$ separately, an estimate of the $85 \%$ threshold from each run was calculated from the respective psychometric function (a cumulative gaussian) on a per-subject basis. The largest of the three modulation depth thresholds was discarded and the mean of the other two was used 
as the threshold depth for that subject in the TMS experiment.

\section{fMRI Acquisition and Analysis}

Subjects wore earplugs underneath the sound-attenuating headphones from the fMRI-compatible auditory stimulus delivery system (MR Confon, MR Confon GmBH). fMRI data were acquired using an Interleaved Silent Steady-State fMRI sequence (Schwarzbauer et al. 2006). The sound level of the scanner noise, not accounting for attenuation provided by earplugs and ear defenders, was $81 \mathrm{~dB}$ SPL during the quiet period and $98 \mathrm{~dB}$ SPL during the acquisition period. During the acquisition periods, whole head fMRI data (GE-EPI, TR $=2 \mathrm{~s}, \mathrm{TE}=$ minimum full, $\mathrm{FA}=90^{\circ}$ ) were collected using a GE Signa HDx 3T system (General Electric, WI, USA). A $128 \times 128$ pixel matrix with a field of view of $25.6 \mathrm{~cm}$ was used, giving an in-plane resolution of $2 \times 2 \mathrm{~mm}$. 24 interleaved slices were collected with a slice thickness of $2 \mathrm{~mm}$. Slices were oriented parallel with the Sylvian fissure while ensuring bilateral coverage of Heschl's gyrus and planum temporale.

Stimulus conditions were silence, noise, $4 \mathrm{~Hz}$ AM noise, and $40 \mathrm{~Hz}$ AM noise. All noise carriers were broadband $(0-8000 \mathrm{~Hz})$ and the AM stimuli were fully modulated $(m=1)$. Stimuli were presented for 6 seconds followed by an acquisition period of 8s. Each stimulus was presented 6 times in each fMRI run and three runs were performed for each subject, each run lasting approximately 6 minutes. Data were analysed using Feat 5.98, part of FSL-4.1 (Smith et al. 2004), along with custom scripts which implemented filtering of the temporally non-contiguous data. Full details of this analysis procedure can be found in Hymers et al. (2015), with the exception that in this study spatial smoothing was performed using a kernel with a FWHM of $2 \mathrm{~mm}$.

Data from the multiple fMRI runs for each subject were combined using a fixedeffects analysis. A contrast of $4 \mathrm{~Hz}$ and $40 \mathrm{~Hz}$ AM noise combined over unmodulated noise was performed. The results were corrected for multiple comparisons using 
a cluster thresholding procedure $(\mathrm{Z}>2.3, \mathrm{p}=0.05$; Worsley 2001). TMS targets were defined individually for each subject using a superficial maxima voxel in a significant cluster on posterior superior temporal gyrus / planum temporale for each hemisphere.

Sagittal whole head structural T1-weighted data (3D FSPGR, TR $=7.8 \mathrm{~ms}$, TE $=$ minimum full, $\mathrm{FA}=20^{\circ}$, Matrix $256 \times 256, \mathrm{FOV}=29.0 \mathrm{~cm}, 176$ slices, Slice thickness $=1 \mathrm{~mm}$ ) were collected in the same scanning session as the fMRI data for participants who did not already have a structural MRI scan available.

\section{TMS}

A 2x2x3 repeated measures design was used during the main TMS experiment. Experimental factors were adaptation $\mathrm{AM}$ rate (2 levels; $4 \mathrm{~Hz}, 40 \mathrm{~Hz})$, test $\mathrm{AM}$ rate $(2$ levels; 4Hz, 40Hz) and TMS (3 levels; sham, left, right). The dependent variable was accuracy on the AM detection task. Test AM rate was randomised within each session, so all subjects completed six TMS sessions, one for each adaptation AM rate by TMS combination. The order of sessions was approximately counterbalanced across subjects using a latin square design. Each TMS session was separated by at least 24 hours. A Brainsight frameless stereotaxic system (Rogue Research, Montreal, Canada) with chin rest and forehead support was used to monitor coil placement and ensure accurate TMS delivery. A Magstim Super Rapid ${ }^{2}$ (Magstim, Whitland, UK) stimulator and a $80 \mathrm{~mm}$ (external diameter of each ring) figure-of-eight coil (Magstim, Whitland, UK) was used for pulse delivery. The TMS coil was oriented with the handle pointing backwards, parallel with the midline. Stimulation consisted of a double-pulse protocol with pulses separated by $50 \mathrm{~ms}$. TMS pulse intensity was set to $65 \%$ of the maximum stimulator output (maximum $=2.6 \mathrm{~T}$ ) based on previous state-dependent TMS studies (Cattaneo and Silvanto 2008; Cattaneo et al. 2008). Before the first experimental TMS session, test pulses were delivered to ensure that subjects could tolerate this TMS protocol. The left and right TMS targets were ob- 
tained using the fMRI data as previously described. Sham TMS was performed by placing the coil at the vertex and rotating by $90^{\circ}$ to remove any physiological effect of TMS but, crucially, retain peripheral factors such as the auditory artefact (Lisanby et al. 2001). Stimuli were delivered during the experiment using the same Etymotic ER-2 headphones and earpieces as were used during psychophysical testing.

A graphical outline of the TMS session can be seen in Figure 1. Every TMS session began with a period of auditory adaptation during which 10 minutes of broadband noise which had been fully amplitude modulated at a rate of either $4 \mathrm{~Hz}$ or $40 \mathrm{~Hz}$ was presented depending on the condition being tested. This period was to ensure participants were maximally adapted (Wojtczak and Viemeister 2005). All further behavioural adaptation within the session was at the same AM rate. A block of 150 trials was then performed. Each trial consisted of 6 seconds of behavioural adaptation followed by a $2 \mathrm{AFC}$ task. A double-pulse TMS burst was delivered during the adaptation sound, immediately prior to the 2AFC task. Each trial perceptually formed one continuous $7.5 \mathrm{~s}$ auditory event. The design of the $2 \mathrm{AFC}$ task was identical to that used during baseline psychophysical testing, aside from the fixed (85\% threshold) AM depth of the target interval. No feedback was given during the TMS sessions. During each block the test AM rate was pseudo-randomised; 75 trials were modulated at a rate of $4 \mathrm{~Hz}$ and the other 75 were modulated at a rate of $40 \mathrm{~Hz}$. At the completion of the first block of trials, 4 minutes of fully modulated AM noise (top-up adaptation) were presented at the same rate used at the start of the experiment to ensure that the adaptation effect was consistent. This period also allowed the participant to have a short break from responding, during which the TMS coil was switched with an identical replacement to ensure the coil remained at an operational temperature. A second block of 150 trials was then completed. 


\section{Statistical Analysis}

Generalised linear mixed-effects models (GLMM) were fit to the TMS accuracy data. This technique accounts for interdependence of the data arising from repeated measurements of the same participants; it also allows adjustments for non-normal distributions (Baayen et al. 2008; Dixon 2008; Jaeger 2008). A logit link function was used to account for the binomial distribution of the raw data. Separate models were fit to the $4 \mathrm{~Hz}$ and $40 \mathrm{~Hz}$ test $\mathrm{AM}$ rate data. Both models included fixed effects of TMS condition and behavioural adaptation condition and an interaction term. Participant was included as a random effect. Random intercepts and slopes for TMS condition and behavioural adaptation condition were estimated for each participant. The random effects structure was the maximal justified by the design (Barr et al. 2013). Treatment coding was used to measure the difference from the baseline condition of sham TMS with $4 \mathrm{~Hz}$ AM adaptation (the baseline condition choice is arbitrary and was chosen to make other parameters easier to interpret). Finally, parametric bootstrapping was conducted on the model parameters to obtain $95 \%$ confidence intervals. All modelling was conducted using the lme4 package (Bates et al. 2015) in $R$ software (R Core Team 2017). Parametric bootstrapping was conducted using the pbkrtest package (Halekoh and Højsgaard 2014). 


\section{Results}

\section{Psychophysics}

The modulation depth at which $85 \%$ accuracy was achieved was calculated individually for each participant, for each AM test rate (4 and $40 \mathrm{~Hz}$ ). The mean modulation depth across participants for the $4 \mathrm{~Hz}$ condition was $-20.7 \mathrm{~dB}$ and for the $40 \mathrm{~Hz}$ condition was $-21.2 \mathrm{~dB}$. A paired t-test showed no significant difference between thresholds for $4 \mathrm{~Hz}$ and $40 \mathrm{~Hz} \mathrm{AM}$ detection $(\mathrm{t}(7)=0.888, \mathrm{p}=0.404)$.

\section{fMRI derivation of TMS Targets}

Figure 2 shows targets in both hemispheres for an example participant.

TMS target distance measurements were recorded for TMS pulses. This is a measure of the distance from the centre of the TMS coil to the fMRI-defined target. Four sessions lacked these measurements due to a technical issue. A linear mixedeffects model was fit to these data, with a fixed effect of TMS hemisphere and a random effect of participant. Parametric bootstrapping of the model parameters was conducted to obtain $95 \%$ confidence intervals. There was a negligible mean difference of $-0.558 \mathrm{~mm}, 95 \%$ CI [-10.025, 8.631] between left hemisphere and right hemisphere conditions. There was no overall correlation between the AM task accuracy scores and the target distance measurements $(\mathrm{r}=0.008, \mathrm{p}=0.954)$.

TMS positioning error measurements were also recorded. This is a measure of the difference between the fMRI-defined target and the estimated pulse trajectory. Four sessions lacked these measurements due to a technical issue. A linear mixedeffects model was fit to these data, with a fixed effect of TMS hemisphere and a random effect of participant. Parametric bootstrapping of the model parameters was conducted to obtain $95 \%$ confidence intervals. There was a negligible mean difference of $-0.031 \mathrm{~mm}, 95 \%$ CI $[-0.082,0.021]$ between left hemisphere and right hemisphere conditions. The overall mean error was $0.342 \mathrm{~mm}, 95 \%$ CI [0.320, 0.364]. 


\section{Sham TMS Behavioural Analysis}

The effects of adaptation without TMS were assessed using the sham TMS conditions. This ensured that any noise from the TMS coil was incorporated into the baseline measure. For the $4 \mathrm{~Hz}$ test condition, a mean accuracy decrease of $26 \%$ was seen when participants were adapted to $4 \mathrm{~Hz}$ compared to when they were adapted to $40 \mathrm{~Hz}$. For the $40 \mathrm{~Hz}$ test condition, a mean accuracy decrease of $22 \%$ was seen when participants were adapted to $40 \mathrm{~Hz}$ compared to when they were adapted to 4 $\mathrm{Hz}$ AM noise. A paired t-test showed no significant difference in level of adaptation in the matched cases; i.e. $4 \mathrm{~Hz}$ adaptor followed by $4 \mathrm{~Hz}$ test versus $40 \mathrm{~Hz}$ adaptor followed by $40 \mathrm{~Hz}$ test $(\mathrm{t}(7)=1.222, \mathrm{p}=0.261)$.

\section{GLMM analyses}

The data were divided based on test AM rate and separate generalised linear mixed effects models with logit link functions (GLMMs) were fit to the two subsets. These models assume that the log-odds of the binomial response variable varies linearly. Results are presented in logits to aid interpretation as confidence intervals are symmetrical about the point estimate and each coefficient value directly represents the effect size. Using logits also preserves the effect direction represented by the sign of the coefficients. The coefficient for the baseline condition represents the overall mean intercept and the other coefficients represent the (population-level) difference from this baseline for each condition. For consistency, the baseline condition for both models was sham TMS with adaptation to $4 \mathrm{~Hz}$ AM.

Whilst assessments of statistical significance using conventional metrics such as p-values are difficult to perform with GLMMs due to the unknown denominator degrees of freedom required to calculate the F-statistic, confidence intervals can be computed using parametric bootstrapping (Halekoh and Højsgaard 2014). These 95\% confidence intervals can be used to approximately assess statistical significance 
at the 0.05 level. If a value of 0 is not contained within the upper and lower bounds of the confidence interval, then the result is approximately statistically significant using a type-I error rate of $5 \%$. While there are clear issues surrounding the use and interpretation of dichotomous assessments of statistical significance (McShane et al. 2017), they are conventional and informative so the (approximately) statistically significant results have been indicated by an asterisk in the results tables.

\section{GLMM analysis of $40 \mathrm{~Hz}$ test rate data}

Results from this model are shown in Table 1 with discussed results indicated by an asterisk. Behavioural adaptation to $40 \mathrm{~Hz} \mathrm{AM}$ had a clear negative effect on the $40 \mathrm{~Hz}$ test rate data, as shown by the negative $40 \mathrm{~Hz}$ Adaptation coefficient. This result replicates previous psychophysical work on selective adaptation to AM (e.g. Wojtczak and Viemeister 2005). TMS to the left hemisphere had a net positive effect on $40 \mathrm{~Hz}$ AM detection over both adaptation rates, as shown by the positive Left Hemisphere TMS coefficient. However, these main effects are confounded by a rate by hemisphere interaction, as seen in Figure 3. The negative Left Hemisphere $T M S \times 40 H z$ Adaptation interaction coefficient represents the differential effect that adaptation to $40 \mathrm{~Hz}$ AM had compared to $4 \mathrm{~Hz}$ adaptation, specifically in the left hemisphere TMS condition. As the baseline condition is $4 \mathrm{~Hz}$ AM with sham TMS, this interaction coefficient represents the net change from sham to left TMS and 4 $\mathrm{Hz}$ adaptation to $40 \mathrm{~Hz}$ adaptation.

\section{GLMM analysis of $4 \mathrm{~Hz}$ test rate data}

Results from this model are shown in Table 2 with discussed results indicated by an asterisk. Accuracy on the $4 \mathrm{~Hz}$ AM detection task was much lower when participants were behaviourally adapted to $4 \mathrm{~Hz}$ AM compared to when participants were 
behaviourally adapted to $40 \mathrm{~Hz} \mathrm{AM}$. This is shown by the positive $40 \mathrm{~Hz}$ Adaptation coefficient; the only coefficient from the $4 \mathrm{~Hz}$ test rate model where the $95 \% \mathrm{CI}$ did not cross zero, as shown in Table 2. This is consistent with the aforementioned psychophysical work that showed peak adaptation for matched adaptation and test rates (Wojtczak and Viemeister 2005). The chosen baseline condition explains the difference in direction for the adaptation effect between the two models; a decrease in accuracy is shown in the $40 \mathrm{~Hz}$ GLMM whereas an increase in accuracy is shown in the $4 \mathrm{~Hz}$ GLMM. The lack of a statistically significant TMS or interaction effect suggests TMS was unable to affect AM processing in the same way as with $40 \mathrm{~Hz}$ processing. 


\section{Discussion}

The present study was designed to investigate whether TMS to auditory areas could affect AM detection performance and if so, whether the induced behavioural changes showed state-dependency and/or provided support for asymmetric accounts of auditory processing. Three specific research questions were posed.

The first question asked whether AM detection could be affected by TMS to auditory cortex. A significant effect of left hemisphere TMS was shown compared to sham TMS, but only in the $40 \mathrm{~Hz}$ AM detection condition.

The second question asked whether TMS effects showed state-dependency, i.e. did behavioural adaptation have an effect on the direction or degree to which TMS modulated performance on the task. In the conditions with left hemisphere TMS and $40 \mathrm{~Hz}$ AM detection, state-dependent TMS effects were found, though the change in behavioural performance was not in the predicted direction; a facilitation of AM detection was predicted in matched adaptation conditions, however in the matched $40 \mathrm{~Hz}$ condition, TMS caused a decrease in AM detection accuracy.

The final question asked whether any TMS effects were consistent with asymmetric models of auditory processing, with directional predictions following the AST hypothesis (Poeppel 2003). Left hemisphere stimulation was predicted to preferentially modulate $40 \mathrm{~Hz}$ AM detection whilst right hemisphere stimulation was predicted to preferentially modulate $4 \mathrm{~Hz} \mathrm{AM}$ detection. Left hemisphere stimulation was shown to affect the detection of $40 \mathrm{~Hz} \mathrm{AM}$, whilst no effect of right hemisphere stimulation was shown on the detection of either 4 or $40 \mathrm{~Hz} \mathrm{AM}$.

\section{Effect of AM rate}

Our results show that whilst TMS to the left hemisphere affected detection of $40 \mathrm{~Hz}$ AM, neither right nor left hemisphere TMS affected detection of $4 \mathrm{~Hz}$ AM. Before considering possible explanations for this difference, some potential confounds must 
be addressed. The number of modulation cycles within the presentation period is known to affect AM detection ability (Sheft and Yost 1990). The 500ms period contained 2 cycles of $\mathrm{AM}$ in the $4 \mathrm{~Hz}$ test condition compared to 20 cycles in the $40 \mathrm{~Hz}$ test condition. To address this possible confound, detection difficulty was calibrated separately for each AM rate and for each participant.

Several studies have shown that BOLD signal changes are both smaller and less distributed in response to higher frequency AM compared to lower frequency AM (Giraud et al. 2000; Harms and Melcher 2002). Therefore, the choice of TMS target locations could also affect whether, and to what degree, processing of different modulation rates would be affected by TMS. Cortical TMS targets were chosen based on fMRI responses to $4 \mathrm{~Hz}$ and $40 \mathrm{~Hz}$ AM combined, contrasted over unmodulated noise to avoid biasing the targets towards areas more specialised for processing either rate.

The observation that TMS only affected $40 \mathrm{~Hz}$ AM detection could be due to the use of a different and possibly more robust mechanism for coding lower rates of modulation (such as $4 \mathrm{~Hz}$ ). Evidence from multiple modalities has converged in support of such an account where low and high frequency AM is coded by different mechanisms. fMRI studies have found that lower frequency AM elicits a more sustained response whilst higher frequency AM produces more phasic responses at onset and offset of the stimulus, possibly representing a single auditory event (Giraud et al. 2000; Harms et al. 2005). Direct cortical recordings using invasive intracranial electrodes have also provided strong support for a shift in coding mechanism as AM rate increases (Brugge et al. 2009; Nourski et al. 2013; Nourski 2017). Non-invasive electrophysiological evidence shows that the balance of phase-locked (PL) and non phase-locked (NPL) activity changes as AM rate increases; PL activity dominates at lower rates whilst more NPL activity is observed with increasing AM rate (Tang et al. 2016). The cutoff point for this transition from mostly PL to mostly NPL activity is estimated to be $50 \mathrm{~Hz}$ (above the rates studied here), but the amount of 
NPL activity does differ between $4 \mathrm{~Hz}$ and $40 \mathrm{~Hz}$. Though PL activity still dominates the response to $40 \mathrm{~Hz}$ AM, Tang et al. (2016) found a significant increase in NPL activity over that measured for $4 \mathrm{~Hz} \mathrm{AM}$. It is possible that this difference in coding mechanisms might contribute to the observed results. Any mechanism which relies on coding the onset and offset of a stimulus could be more susceptible to TMS than one reliant on sustained responses. If, for example, the single cortical event coding for the onset of $40 \mathrm{~Hz}$ AM was disrupted, this may have a significant impact, particularly at short stimulus durations in which there is little time for the system to recover. In comparison, a sustained system where PL neurons fire in synchronisation with slower modulations would likely be more robust to a single disruptive event. Further, it would be biologically useful for the system that processes low-frequency AM it to be more robust to disruption due to its importance for speech intelligibility. This explanation is challenged however, by the classic finding that with sustained periods of auditory stimulation, peak PL responses are shown for $40 \mathrm{~Hz} \mathrm{AM}$; the $40 \mathrm{~Hz}$ auditory steady-state response (Galambos et al. 1981; Ross et al. 2000). Though PL and NPL activity is evident during $40 \mathrm{~Hz}$ processing, the role and relative importance of each mechanism is not clear. The suggestion that TMS may differentially affect disparate neuronal coding mechanisms has important implications for the design of future TMS experiments and warrants further investigation.

\section{Hemispheric differences}

Before considering explanations of the changes in behavioural performance caused by TMS to the left and right hemisphere, we can first rule out several methodological confounds. Neither the distance to the TMS target, nor the accuracy with which TMS was delivered differed significantly between the two hemispheres or between test conditions. In addition, there was no significant correlation across subjects between task accuracy scores and the distance to target, suggesting that any distance effects can be discounted. 
Previous research using transcranial direct current stimulation (TDCS) to investigate auditory temporal resolution using a gap detection task has shown differential susceptibility of the left and right hemispheres to stimulation. A detrimental effect on performance was found exclusively when anodal TDCS was applied to the left hemisphere (Heimrath et al. 2014). It is interesting that the current results accord with this finding as there is evidence that gap detection tasks and estimation of the temporal modulation transfer function (TMTF; using the method employed in this study, though the full TMTF was not estimated here) are measuring different aspects of auditory temporal acuity (Shen 2014). Disruptive TMS effects on duration discrimination have been shown exclusively for the right hemisphere (Bueti et al. 2008). However, these studies differ not only in both the nature of the behavioural task, but also the nature of the stimulus. Whilst Bueti et al. (2008) used tonal stimuli, Heimrath et al. (2014) and the current study use relatively broadband noise stimuli. A question of interest is therefore whether the spectral nature of the stimulus has an influence on hemispheric lateralisation of function and how this interacts with the specific behavioural task under investigation.

\section{State-dependent effects}

The initial prediction for this study was that TMS combined with behavioural adaptation at a modulation rate matched to the test modulation rate would lead to an improvement in AM detection accuracy. Instead, an unexpected dissociation was shown between performance on the $40 \mathrm{~Hz}$ AM detection task in the matched and un-matched adaptation conditions: a behavioural facilitation was observed in the unmatched condition (4 Hz adaptation) whilst a reduction in performance was seen in the matched condition (40 Hz adaptation). An overarching framework that accounts for both online and state-dependent effects has recently been proposed (Silvanto and Cattaneo 2017). It is possible to reconcile these results within this framework, however not using a purely state-dependent TMS explanation. This 
suggests that modifications made to the experimental design when converting it to investigate auditory function may have affected the nature of the disruption. The majority of state-dependent TMS experiments have used visual tasks involving brief simultaneous presentations of multiple stimuli with TMS pulses delivered on stimulus onset (Silvanto et al. 2007; Cattaneo and Silvanto 2008; Silvanto and Muggleton 2008). To account for the necessarily more temporally extended nature of auditory stimuli, modifications were required when designing this auditory state-dependent TMS paradigm. In each trial, an auditory adaptation period immediately preceded a 2-AFC decision, with TMS delivered just prior to the onset of the 2-AFC task. In matched adaptation trials, though the stimulation is delivered whilst the participant is adapted, it is actually delivered during AM processing at the target rate, therefore corresponding more closely to online TMS. The suprathreshold TMS would then be expected to impair performance as the neurons tuned to the target rate have been shifted into their inhibitory range (Silvanto and Cattaneo 2017: fig.1). This key timing difference could explain the 'virtual lesion' style detrimental TMS effects seen in the matched $40 \mathrm{~Hz}$ condition.

In unmatched adaptation trials, participants received TMS during processing of one rate of $\mathrm{AM}$ and were subsequently tested at the other rate. Within this same overarching framework, two possible explanations exist that lead to a facilitation of behavioural performance. At the point at which TMS was delivered, neural populations responsible for processing the test rate would be suppressed relative to populations responsible for processing the adaptation rate. This would lead to test rate populations being in their facilitatory range when activated by the suprathreshold TMS, leading to behavioural improvements at detecting 40Hz AM (Silvanto and Cattaneo 2017: fig. 2). Alternatively, It is also possible that the neural populations that process $40 \mathrm{~Hz}$ AM may have been adapted by the $4 \mathrm{~Hz}$ AM. Maximal adaptation, as measured by decrease in detection ability, occurs when adaptor and test rates are 
identical, however there is evidence for a graded decrease in adaptation as the difference between the two rates increases (Houtgast 1989; Bacon and Grantham 1989; Wojtczak and Viemeister 2005). Based on the estimated shape of the modulation filter with centre frequency of $40 \mathrm{~Hz}$, it may be expected that populations involved in processing $40 \mathrm{~Hz}$ would be adapted by exposure to $4 \mathrm{~Hz}$ AM. Further, the estimated shape of modulation filters with centre frequencies of 4 and $40 \mathrm{~Hz}$ differs such that the $4 \mathrm{~Hz}$ filter is much more narrow ( $5 \mathrm{~Hz}$ bandwidth), suggesting an asymmetry where populations optimised for coding $4 \mathrm{~Hz}$ would not be adapted by exposure to $40 \mathrm{~Hz}$ AM, but populations optimised for coding $40 \mathrm{~Hz}$ may still be adapted somewhat by exposure to $4 \mathrm{~Hz}$ AM (Dau et al. 1997b). Under this assumption, the outcome in the unmatched condition would follow state-dependent TMS predictions, where adaptation has suppressed the activity of $40 \mathrm{~Hz}$ populations, shifting the inhibitory/facilitatory range of these populations such that suprathreshold TMS leads to behavioural facilitation. With the current dataset, it is not possible to dissociate these two mechanisms, both of which would lead to an increase in performance for $40 \mathrm{~Hz} \mathrm{AM}$ detection.

Overall, the timing of TMS delivery is central to this explanation of the observed results. The importance of this parameter has been previously noted (Silvanto and Pascual-Leone 2008), with differential effects shown when pulses were delivered before and during task performance across different studies, with and without adaptation. In online TMS studies, no adaptation is used and suprathreshold TMS is delivered during the test stimulus, causing a decrease in performance. The current study therefore demonstrates that TMS can cause either disruptive or facilitatory effects on $40 \mathrm{~Hz}$ AM detection depending on which AM rate is perceived during TMS delivery. Further investigating how TMS at different timepoints affects AM detection will help to elucidate the mechanisms of this effect and improve the utility of auditory TMS as an investigative tool. 


\section{${ }_{512}$ Conclusions}

This study showed, for the first time, that low-level mechanisms involved in AM detection, a key component of speech processing, can be affected using TMS. This important finding demonstrates that TMS can be a useful technique for investigating low-level auditory processing mechanisms and opens the door to new research in this area. It was also shown that the TMS effect on AM detection shows statedependency, i.e., the underlying state of the AM detection system when TMS is delivered has a differential effect on the outcome. It was further demonstrated that systematically modifying this underlying state using behavioural adaptation is an effective way to increase the functional resolution of TMS and alter the nature of TMS effects on AM detection. The results of this study provide support for the importance of the left posterior superior temporal gyrus for processing $40 \mathrm{~Hz} \mathrm{AM}$. It also suggests that $4 \mathrm{~Hz}$ AM may be encoded using a different mechanism that is more robust to interference from TMS. This illustrates a potential limitation of the method and would benefit from further investigation. Finally, in addition to the direct implications for auditory processing, methodological considerations for the use and interpretation of state-dependent TMS were discussed so that important factors such as subtle timing and task manipulations are accounted for in future work. This study demonstrated the utility of TMS as a new and potentially valuable tool for furthering understanding of low-level auditory processing in health and disease.

\section{Acknowledgments}

AMP is supported by a PhD studentship from the Economic and Social Research Council (grant number: ES/J500215/1). The authors would like to acknowledge assistance from Faith Marsh, Elenor Morgenroth and Sofia Tsitsopoulou with the collection of the data as part of their MSc empirical research project. 


\section{References}

Andoh J, Zatorre RJ (2011) Interhemispheric connectivity influences the degree of modulation of TMS-induced effects during auditory processing. Frontiers in Psychology 2:161.

Baayen RH, Davidson DJ, Bates DM (2008) Mixed-effects modeling with crossed random effects for subjects and items. Journal of Memory and Language 59:390-412.

Bacon SP, Grantham DW (1989) Modulation masking: Effects of modulation frequency, depth, and phase. The Journal of the Acoustical Society of America $85: 2575-2580$.

Barr DJ, Levy R, Scheepers C, Tily HJ (2013) Random effects structure for confirmatory hypothesis testing: Keep it maximal. Journal of Memory and Language 68:255-278.

Barton B, Venezia JH, Saberi K, Hickok G, Brewer AA (2012) Orthogonal acoustic dimensions define auditory field maps in human cortex. Proceedings of the National Academy of Sciences 109:20738-20743.

Bates DM, Maechler M, Bolker B, Walker S (2015) Fitting linear mixed-effects models using lme4. Journal of Statistical Software 67:1-48.

Brugge JF, Nourski KV, Oya H, Reale Ra, Kawasaki H, Steinschneider M, Howard Ma (2009) Coding of repetitive transients by auditory cortex on Heschl's gyrus. Journal of Neurophysiology 102:2358-2374.

Bueti D, van Dongen EV, Walsh V (2008) The role of superior temporal cortex in auditory timing. PLoS ONE 3:e2481.

Cattaneo Z, Rota F, Vecchi T, Silvanto J (2008) Using state-dependency of transcranial magnetic stimulation (TMS) to investigate letter selectivity in the left 
posterior parietal cortex: A comparison of TMS-priming and TMS-adaptation paradigms. European Journal of Neuroscience 28:1924-1929.

Cattaneo Z, Silvanto J (2008) Time course of the state-dependent effect of transcranial magnetic stimulation in the TMS-adaptation paradigm. Neuroscience Letters 443:82-85.

Dau T, Kollmeier B, Kohlrausch A (1997a) Modeling auditory processing of amplitude modulation. II. Spectral and temporal integration. The Journal of the Acoustical Society of America 102:2906-2919.

Dau T, Kollmeier B, Kohlrausch A (1997b) Modeling auditory processing of amplitude modulation. I. Detection and masking with narrow-band carriers. The Journal of the Acoustical Society of America 102:2892.

Dixon P (2008) Models of accuracy in repeated-measures designs. Journal of Memory and Language 59:447-456.

Drullman R, Festen JM, Plomp R (1994a) Effect of reducing slow temporal modulations on speech reception. The Journal of the Acoustical Society of America 95:2670.

Drullman R, Festen JM, Plomp R (1994b) Effect of temporal envelope smearing on speech reception. The Journal of the Acoustical Society of America 95:1053.

Edwards E, Chang EF (2013) Syllabic ( 2-5 Hz) and fluctuation $(\sim 1-10 \mathrm{~Hz})$ ranges in speech and auditory processing. Hearing Research 305:113-134.

Galambos R, Makeig S, Talmachoff PJ (1981) A 40-Hz auditory potential recorded from the human scalp. Proceedings of the National Academy of Sciences 78:2643-2647.

Giraud AL, Lorenzi C, Ashburner J, Wable J, Johnsrude I, Frackowiak RSJ, 
Kleinschmidt A (2000) Representation of the temporal envelope of sounds in the human brain. Journal of Neurophysiology 84:1588-1598.

Halekoh U, Højsgaard S (2014) A Kenward-Roger Approximation and Parametric Bootstrap Methods for Tests in Linear Mixed Models - The R Package pbkrtest. Journal of Statistical Software 59:1-32.

Harms MP, John GJ, Sigalovsky IS, Melcher JR (2005) Short-term sound temporal envelope characteristics determine multisecond time patterns of activity in human auditory cortex as shown by fMRI. Journal of Neurophysiology 93:210-222.

Harms MP, Melcher JR (2002) Sound repetition rate in the human auditory pathway: representations in the waveshape and amplitude of fMRI activation. Journal of Neurophysiology 88:1433-1450.

Heimrath K, Kuehne M, Heinze HJ, Zaehle T (2014) Transcranial direct current stimulation (tDCS) traces the predominance of the left auditory cortex for processing of rapidly changing acoustic information. Neuroscience 261:68-73.

Herdener M, Esposito F, Scheffler KR, Schneider P, Logothetis NK, Uludag K, Kayser C (2013) Spatial representations of temporal and spectral sound cues in human auditory cortex. Cortex 49:2822-2833.

Houtgast T (1989) Frequency selectivity in amplitude-modulation detection. The Journal of the Acoustical Society of America 85:1676-1680.

Hymers M, Prendergast G, Liu C, Schulze A, Young ML, Wastling SJ, Barker GJ, Millman RE (2015) Neural mechanisms underlying song and speech perception can be differentiated using an illusory percept. NeuroImage 108:225-33.

Jaeger TF (2008) Categorical data analysis: Away from ANOVAs (transformation or not) and towards logit mixed models. Journal of Memory and Language 59:434-446. 
Jepsen ML, Ewert SD, Dau T (2008) A computational model of human auditory signal processing and perception. The Journal of the Acoustical Society of America 124:422-438.

Kleinschmidt DF, Jaeger TF (2015) Re-examining selective adaptation: Fatiguing feature detectors, or distributional learning? Psychonomic Bulletin \& Review 23:678-691.

Kohlrausch A, Fassel R, Dau T (2000) The influence of carrier level and frequency on modulation and beat-detection thresholds for sinusoidal carriers. The Journal of the Acoustical Society of America 108:723.

Levitt H (1971) Transformed up-down methods in psychoacoustics. The Journal of the Acoustical Society of America 49:467-477.

Lisanby SH, Gutman D, Luber B, Schroeder C, Sackeim HA (2001) Sham TMS: Intracerebral measurement of the induced electrical field and the induction of motor-evoked potentials. Biological Psychiatry 49:460-463.

McGettigan C, Scott SK (2012) Cortical asymmetries in speech perception: what's wrong, what's right, and what's left? Trends in Cognitive Sciences 16:269-276.

McShane BB, Gal D, Gelman A, Robert C, Tackett JL (2017) Abandon statistical significance. arXiv:1709.07588 [stat] arXiv: 1709.07588.

Nourski KV (2017) Auditory processing in the human cortex: An intracranial electrophysiology perspective. Laryngoscope Investigative Otolaryngo$\log y 2: 147-156$.

Nourski KV, Brugge JF, Reale Ra, Kovach CK, Oya H, Kawasaki H, Jenison RL, Howard Ma (2013) Coding of repetitive transients by auditory cortex on posterolateral superior temporal gyrus in humans: an intracranial electrophysiology study. Journal of Neurophysiology 109:1283-95. 
Partridge AM (2017) fMRI-Guided State Dependent TMS of Auditory Cortex: Pre-registration document. doi:10.17605/OSF.IO/FYJMZ .

Peirce JW (2007) PsychoPy — Psychophysics software in Python. Journal of Neuroscience Methods 162:8-13.

Poeppel D (2003) The analysis of speech in different temporal integration windows: Cerebral lateralization as 'Asymmetric Sampling in Time'. Speech Communication 41:245-255.

R Core Team (2017) R: A Language and Environment for Statistical Computing R Foundation for Statistical Computing, Vienna, Austria.

Rees A, Green G, Kay R (1986) Steady-state evoked responses to sinusoidally amplitude-modulated sounds recorded in man. Hearing Research 23:123-133.

Regan D, Tansley BW (1979) Selective adaptation to frequency-modulated tones: evidence for an information-processing channel selectively sensitive to frequency changes. The Journal of the Acoustical Society of America 65:1249-57.

Romei V, Thut G, Silvanto J (2016) Information-based approaches of noninvasive transcranial brain stimulation. Trends in Neurosciences 39:782-795.

Rosen S (1992) Temporal information in speech: Acoustic, auditory and linguistic aspects. Philosophical Transactions of the Royal Society B: Biological Sciences 336:367-373.

Ross B, Borgmann C, Draganova R, Roberts LE, Pantev C (2000) A high-precision magnetoencephalographic study of human auditory steady-state responses to amplitude-modulated tones. The Journal of the Acoustical Society of America 108:679-691. 
Rufener KS, Zaehle T, Oechslin MS, Meyer M (2016) 40 Hz - transcranial alternating current stimulation (tACS) selectively modulates speech perception. International Journal of Psychophysiology 101:18-24.

Schwarzbauer C, Davis MH, Rodd JM, Johnsrude I (2006) Interleaved silent steady state (ISSS) imaging: A new sparse imaging method applied to auditory fMRI. NeuroImage 29:774-782.

Shannon RV, Zeng FG, Kamath V, Wygonski J, Ekelid M (1995) Speech recognition with primarily temporal cues. Science 270:303-304.

Sheft S, Yost WA (1990) Temporal integration in amplitude modulation detection. The Journal of the Acoustical Society of America 88:796-805.

Shen Y (2014) Gap detection and Temporal Modulation Transfer Function as behavioral estimates of auditory temporal acuity Using band-limited stimuli in young and older adults. Journal of Speech, Language, and Hearing Research 57:2280-2292.

Silvanto J (2008) State-dependency of transcranial magnetic stimulation. Brain topography 21:1-10.

Silvanto J, Cattaneo Z (2017) Common framework for "virtual lesion" and statedependent TMS: The facilitatory/suppressive range model of online TMS effects on behavior. Brain and Cognition 119:32-38.

Silvanto J, Muggleton NG (2008) Testing the validity of the TMS state-dependency approach: Targeting functionally distinct motion-selective neural populations in visual areas V1/V2 and V5/MT+. NeuroImage 40:1841-1848.

Silvanto J, Muggleton NG, Cowey A, Walsh V (2007) Neural adaptation reveals state-dependent effects of transcranial magnetic stimulation. European Journal of Neuroscience 25:1874-1881. 
Silvanto J, Pascual-Leone A (2008) State-dependency of transcranial magnetic stimulation. Brain Topography 21:1-10.

Simpson AJR, Reiss JD, McAlpine D (2013) Tuning of human modulation filters is carrier-frequency dependent. PLoS ONE 8:e73590.

Smith SM, Jenkinson M, Woolrich MW, Beckmann CF, Behrens TEJ, JohansenBerg H, Bannister PR, De Luca M, Drobnjak I, Flitney DE, Niazy RK, Saunders J, Vickers J, Zhang Y, De Stefano N, Brady JM, Matthews PM (2004) Advances in functional and structural MR image analysis and implementation as FSL. NeuroImage 23:S208-S219.

Tang H, Crain S, Johnson BW (2016) Dual temporal encoding mechanisms in the human auditory cortex: Evidence from MEG and EEG. NeuroImage 128:32-43.

Tansley BW, Suffield JB (1983) Time course of adaptation and recovery of channels selectively sensitive to frequency and amplitude modulation. The Journal of the Acoustical Society of America 74:765.

Viemeister NF (1979) Temporal modulation transfer functions based upon modulation thresholds. The Journal of the Acoustical Society of America 66:1364-1380.

Weisz N, Steidle L, Lorenz I (2012) Formerly known as inhibitory: Effects of 1-Hz rTMS on auditory cortex are state-dependent. European Journal of Neuroscience 36:2077-2087.

Wojtczak M, Viemeister NF (2005) Forward masking of amplitude modulation: basic characteristics. The Journal of the Acoustical Society of America 118:3198-3210.

Worsley K (2001) Statistical analysis of activation images. Functional MRI: An introduction to methods 14:251-270. 
Xiang J, Poeppel D, Simon JZ (2013) Physiological evidence for auditory modulation filterbanks: Cortical responses to concurrent modulations. JASA Express Letters 133:EL7.

Zatorre RJ, Belin P (2001) Spectral and temporal processing in human auditory cortex. Cerebral Cortex 11:946-53.

Zatorre RJ, Belin P, Penhune VB (2002) Structure and function of auditory cortex: Music and speech. Trends in Cognitive Sciences 6:37-46. 


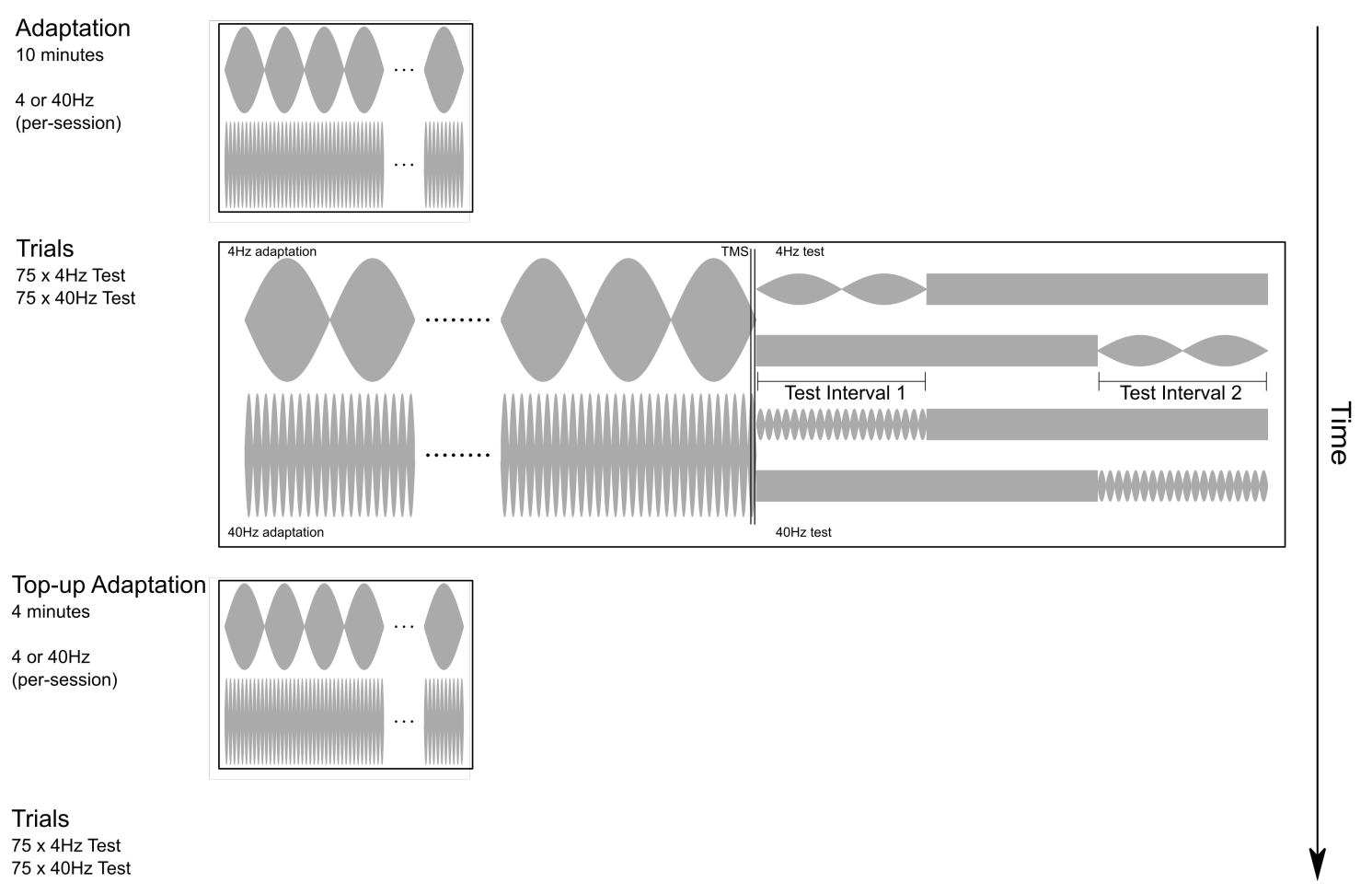

Figure 1: TMS session and trial layout. Time during the session runs from top to bottom. A given session used either a $4 \mathrm{~Hz}$ or $40 \mathrm{~Hz}$ behavioural adaptation stimuli and one of three TMS targets (sham, left or right). Each session began with the subject listening to 10 minutes of fully modulated noise at the session adaptation rate. A block of 150 experimental trials was then performed; 75 test trials were modulated at $4 \mathrm{~Hz}$ and the remaining 75 at $40 \mathrm{~Hz}$. Each trial consisted of $6 \mathrm{~s}$ of adaptation noise (at the rate chosen for the session). Dual-pulse TMS stimulation was locked to the end of the trial adaptation stimulus. A test trial immediately followed the TMS pulses and consisted of a 2-AFC task - subjects were asked to select which of two intervals contained an AM noise. The AM depth was set based upon the initial psychophysical data for the subject. After 150 trials, subjects listened to four minutes of "top-up" adaptation noise. This was followed by a second block of 150 experimental trials of identical layout to the first block. 


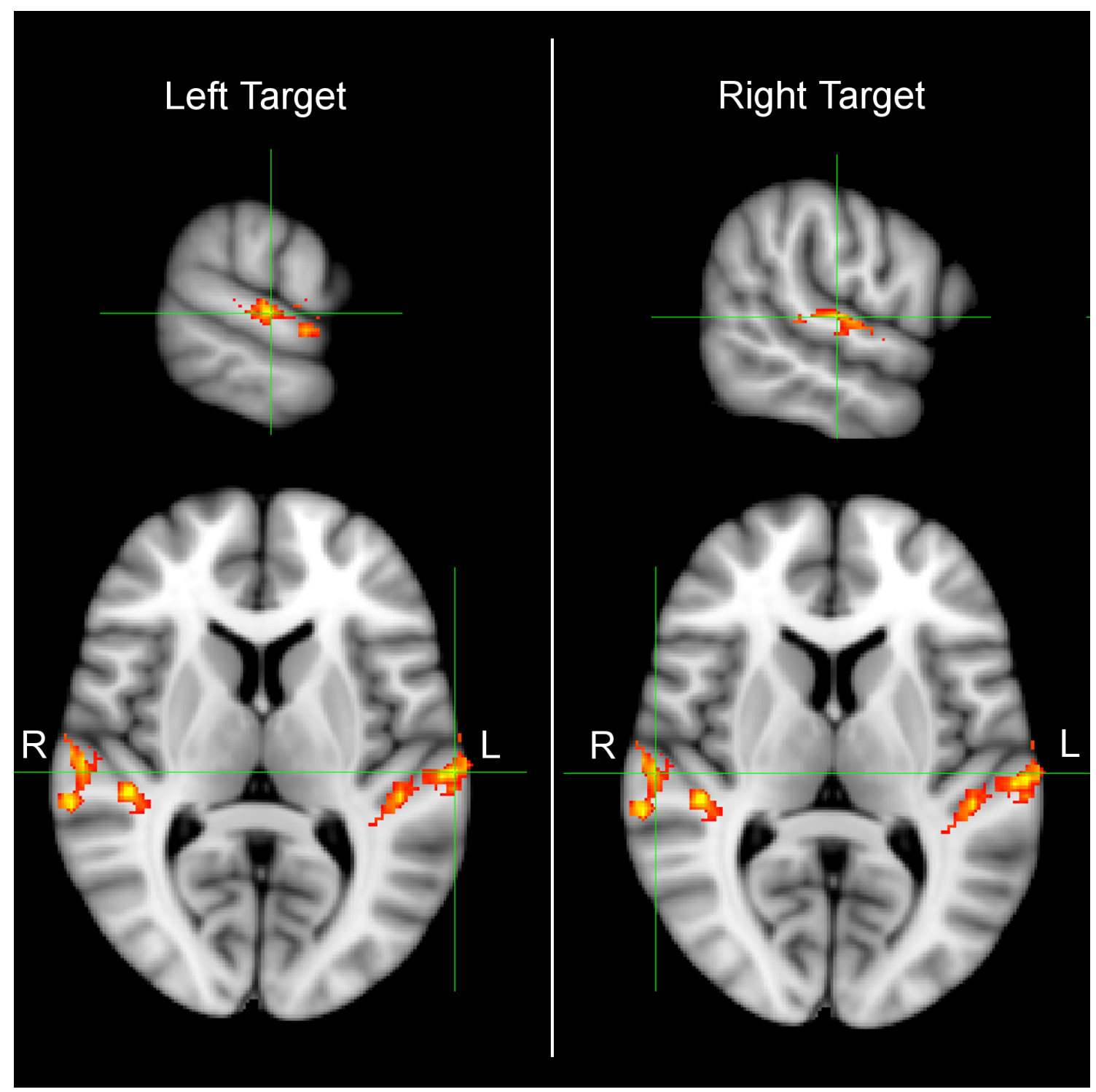

Figure 2: fMRI TMS target localisation data from an example participant. Overlay shows statistically significant clusters of activation $(\mathrm{Z}>2.3 ; \mathrm{p}<0.05)$ in bilateral pSTG/PT in response to a contrast of 4 and $40 \mathrm{~Hz}$ sinusoidally amplitude modulated broadband noise conditions (combined) over unmodulated noise. TMS targets are indicated by the crosshairs. Functional data is shown here on the MNI-152 template brain - targets were transformed back into individual participant co-ordinate space. Target locations for this participant in MNI-152 co-ordinate space are: Left: $-64,-22,8$ and Right: $60,-20,8$ 


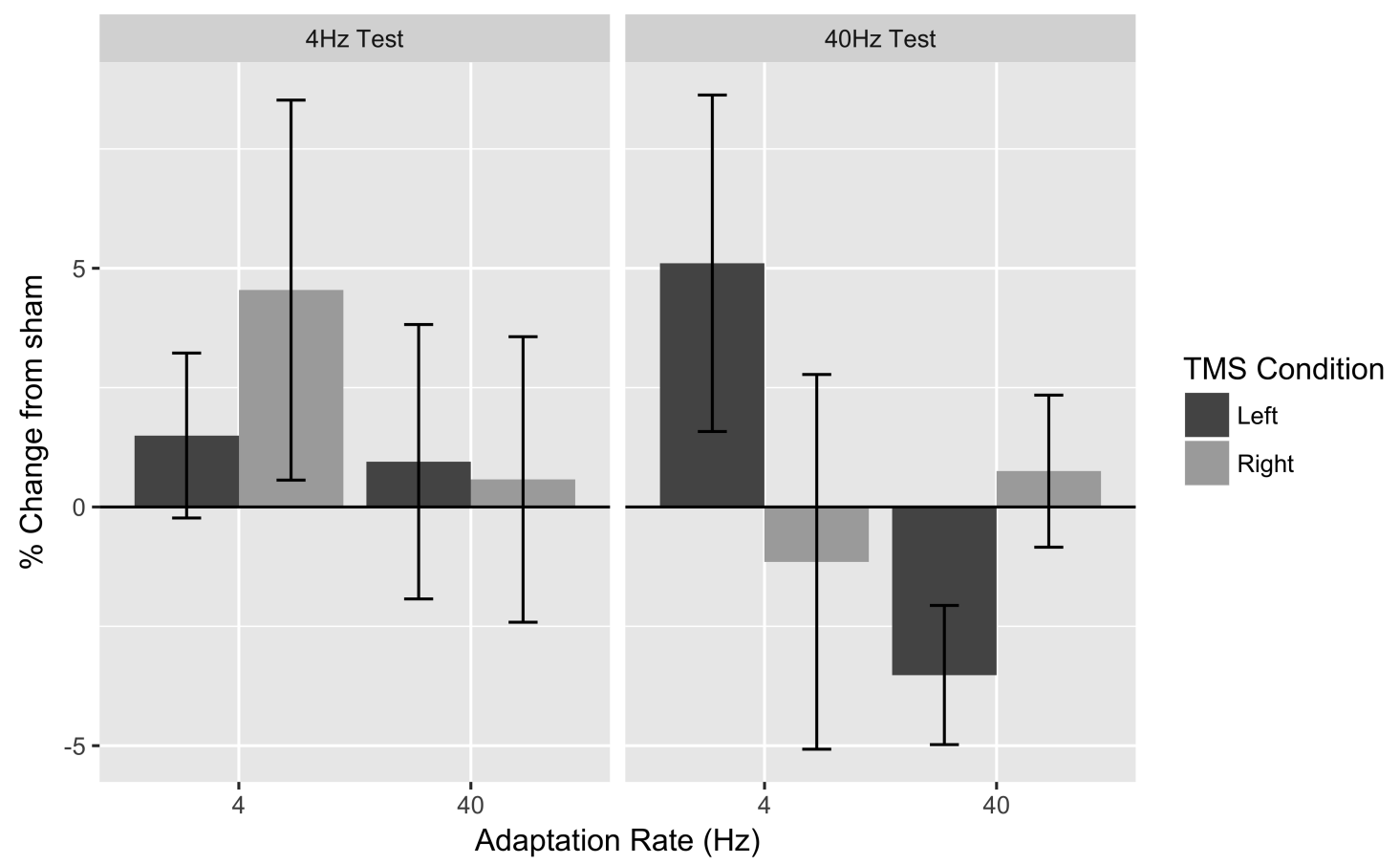

Figure 3: Difference in raw AM detection scores between sham TMS and left or right hemisphere TMS conditions are expressed here as percentage changes. Each bar represents the mean within-subject difference across all participants. Error bars represent the $95 \%$ confidence interval. 


\begin{tabular}{lrrrrl}
\hline & \multicolumn{4}{c}{ Dependent variable: } \\
\cline { 2 - 5 } & Accuracy & \multicolumn{2}{c}{$95 \%$ CI } & & \\
\hline Baseline & 1.018 & 0.669 & 1.378 & \\
Left Hemisphere TMS & 0.302 & 0.096 & 0.519 & $*$ \\
Right Hemisphere TMS & -0.068 & -0.353 & 0.197 & \\
40Hz Adaptation & -1.014 & -1.371 & -0.663 & $*$ \\
Left Hemisphere TMS x 40Hz Adaptation & -0.446 & -0.712 & -0.202 & $*$ \\
Right Hemisphere TMS x 40Hz Adaptation & 0.099 & -0.154 & 0.356 & \\
\hline
\end{tabular}

Table 1: GLMM results for the $40 \mathrm{~Hz}$ AM test conditions. Accuracy values and 95\% CIs are in logit units. 'Baseline' represents the mean in the baseline condition of sham TMS with $4 \mathrm{~Hz}$ behavioural adaptation. All other coefficients represent the difference from this baseline, i.e. the effect size. For example, the $40 \mathrm{~Hz}$ Adaptation co-efficient shows the effect of $40 \mathrm{~Hz}$ behavioural adaptation compared with the baseline of $4 \mathrm{~Hz}$ behavioural adaptation; in this case ( $40 \mathrm{~Hz}$ test trials), the co-efficient shows the expected negative direction, indicating a decrease in AM detection accuracy when participants were behaviourally adapted to $40 \mathrm{~Hz}$. An asterisk is used to mark results discussed in the main text. 


\begin{tabular}{lrrrr}
\hline & \multicolumn{3}{c}{ Dependent variable: } \\
\cline { 2 - 5 } & Accuracy & \multicolumn{2}{c}{$95 \%$ CI } & \\
\hline Baseline & 0.438 & 0.182 & 0.719 & \\
Left Hemisphere TMS & 0.068 & -0.167 & 0.298 & \\
Right Hemisphere TMS & 0.205 & -0.036 & 0.422 & \\
40Hz Adaptation & 1.437 & 1.195 & 1.679 & $*$ \\
Left Hemisphere TMS x 40Hz Adaptation & 0.023 & -0.293 & 0.310 & \\
Right Hemisphere TMS x 40Hz Adaptation & -0.137 & -0.434 & 0.147 & \\
\hline
\end{tabular}

Table 2: GLMM results for the $4 \mathrm{~Hz}$ AM test conditions. Accuracy values and $95 \%$ CIs are in logit units. 'Baseline' represents the mean in the baseline condition of sham TMS with $4 \mathrm{~Hz}$ behavioural adaptation. All other coefficients represent the difference from this baseline, i.e. the effect size. For example, the $40 \mathrm{~Hz}$ Adaptation co-efficient shows the effect of $40 \mathrm{~Hz}$ behavioural adaptation compared with the baseline of $4 \mathrm{~Hz}$ behavioural adaptation; in this case (4Hz test trials), the co-efficient shows the expected positive direction, indicating an increase in AM detection accuracy when participants were behaviourally adapted to $40 \mathrm{~Hz}$. An asterisk is used to mark results discussed in the main text. 